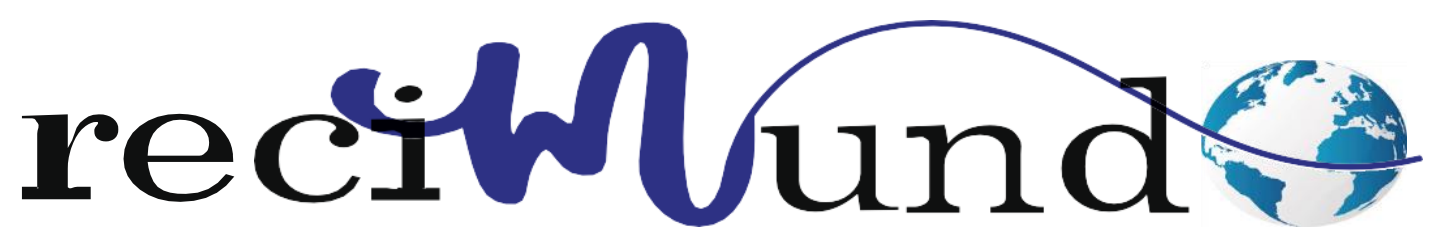

Revista Científica Mundo de la Investigación y el Conocimiento

DOI: $10.26820 /$ recimundo/4.(4).noviembre.2020.216-227

URL: http://recimundo.com/index.php/es/article/view/941

EDITORIAL: Saberes del Conocimiento

REVISTA: RECIMUNDO

ISSN: 2588-073X

TIPO DE INVESTIGACIÓN: Reporte de caso

CÓDIGO UNESCO: 32 Ciencias Médicas

PAGINAS: 216-227

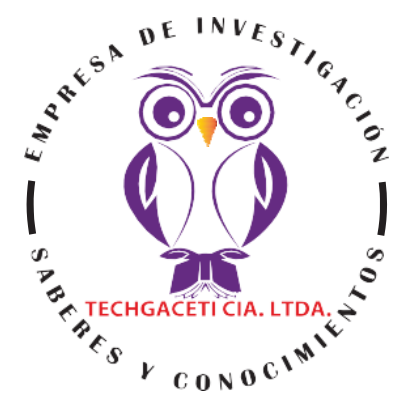

\title{
Coriorretinopatía serosa central crónica. En época de pandemia por covid-19. Serie de casos
}

Chronic central serous chorioretinopathy. In time of a covid-19 pandemic. Number of cases

Coriorretinopatia serosa central crônica. Em tempo de uma pandemia covid-19.

Número decasos

Robinson Barrientos Ortiz'; Ana Luisa González Méndez²

RECIBIDO: 31/08/2020 ACEPTADO: 02/09/2020 PUBLICADO: 10/11/2020

1. Especialista en Oftalmología; Alta especialidad en Retina y Vítreo; Alta especialidad en Microcirugía del Segmento Anterior; Instituto de la Visión. Clínica y Cirugía ocular; Quevedo, Ecuador; robinbarrientos07@gmail.com; (D) 0000-00025085-160k

2. Especialista en Oftalmología; Fellowship de Investigación en Glaucoma; Fellowship de Segmento Anterior; Córnea y Cirugía Refractiva; Clínica La Luz, Lima, Perú; dra.analuisagonzalez88@gmail.com; (D) 0000000298211345

\section{CORRESPONDENCIA}

Robinson Barrientos Ortiz

robinbarrientos07@gmail.com

Quevedo, Ecuador 


\section{RESUMEN}

Objetivo: Demostrar la fluctuación clínica de 3 pacientes con coriorretinopatía serosa central crónica. Métodos: Reporte de casos observacionales de coriorretinopatía serosa central crónica, en un periodo de seguimiento de noviembre 2019-abril 2020. Se incluyeron las variables: revisión de historia clínica, fotografías, tomografía de coherencia óptica de dominio espectral y angiografía fluoresceínica. Resultados: -Masculino de 46 años, clínica de 8 meses con antecedentes personales atópicos y familiares de CSC crónica. AVMC OD. 20/20 OI. 20/32 Segmento anterior normal. Oftalmoscopia OI: área localizada de líquido subretiniano macular. -Masculino de 53 años, con antecedentes atópicos, personalidad tipo A. Clínica de 6 meses con uso inadecuado de esteroides tópicos y vía oral durante su evolución. AVMC OD. 20/25 OI. 20/50. En Ol presenta área localizada parafoveal nasal de líquido subretiniano resto examen visual normal. - Masculino de 40 años, clínica de 7 meses con antecedente de personalidad tipo A. AVMC OD. 20/20 OI. 20/25. En Ol con alteraciones del epitelio pigmentario retiniano macular resto sin novedad clínica. Se inició (diclofenaco $0.1 \%$ ®), (dorzolamida $2 \%+$ timolol $0.5 \%(\AA)$ tópico y espironolactona $50 \mathrm{mg}$ vía oral diario en todos los pacientes. Conclusiones: La CSC es aún una afección enigmática debido en gran parte a una evolución natural de mejoría espontánea en una proporción alta de los pacientes. Ningún tratamiento único ha aportado pruebas abrumadoras de eficacia en los estudios clínicos aleatorizados publicados. Con mayor auge en la era de pandemia por COVID-19 al tener su patogenia relación con el estrés, favorecería el agravamiento de la clínica.

Palabras clave: Coriorretinopatía Serosa Central, Desprendimiento de Retina Seroso, Covid-19, Dorzolamida, Timolol, Diclofenaco, Espironolactona

\section{ABSTRACT}

Objective: To demonstrate the clinical fluctuation of 3 patients with chronic central serous chorioretinopathy. Methods: Report of observational cases of chronic central serous chorioretinopathy, in a follow-up period of November 2019-April 2020. Variables were included: medical history review, photographs, spectral domain optical coherence tomography and fluorescein angiography. Results: - 46-year-old male, 8-month-old clinic with a personal and family history of chronic CSC. BCVA RE. 20/20 LE. 20/32 Normal anterior segment. LE ophthalmoscopy: localized area of macular subretinal fluid. -53-year-old male, with atopic history, type A personality. 6-month clinic with inappropriate use of topical and oral steroids during his evolution. BCVA RE. 20/25 LE. 20/50. In LE there was a localized parafoveal nasal area of subretinal fluid, rest of normal visual examination. - 40-year-old male, 7-month-old clinic with a history of type A personality. BCVA RE. 20/20 LE. 20/25. In LE with alterations of the macular retinal pigment epithelium, the rest were without clinical news. Topical (diclofenac $0.1 \% \AA$ ), (dorzolamide $2 \%$ + timolol $0.5 \% \AA$ ) and spironolactone $50 \mathrm{mg}$ orally daily were started in all patients. Conclusions: CSC is still an enigmatic condition due in large part to a natural history of spontaneous improvement in a high proportion of patients. No single treatment has provided overwhelming evidence of efficacy in published randomized clinical trials. With the greatest boom in the era of the COVID-19 pandemic, as its pathogenesis is related to stress, it would favor the worsening of the clinic.

Keywords: Central Serous Chorioretinopathy, Serous Retinal Detachment, Covid-19, Dorzolamide, Timolol, Diclofenac, Spironolactone

\section{RESUMO}

Objetivo: Demonstrar a flutuação clínica de 3 pacientes com coriorretinopatia serosa central crônica. Métodos: Relato de casos observacionais de coriorretinopatia serosa central crônica, em seguimento de novembro de 2019 a abril de 2020. Foram incluídas as variáveis: revisão do histórico médico, fotografias, tomografia de coerência óptica de domínio espectral e angiografia com fluoresceína. Resultados: - Clínico masculino, 46 anos, 8 meses, com história pessoal e familiar de CSC crônica. BCVA RE. 20/20 LE. 20/32 Segmento anterior normal. Oftalmoscopia LE: área localizada de líquido macular sub-retiniano. Homem de 53 anos, com história atópica, personalidade tipo A. Clínica de 6 meses com uso inadequado de corticoide tópico e oral durante sua evolução. BCVA RE. 20/25 LE. 20/50. Em OE havia área parafoveal nasal localizada de líquido sub-retiniano, resto do exame visual normal. - Clínica do sexo masculino, 40 anos, 7 meses, com história de personalidade tipo A. BCVA RE. 20/20 LE. 20/25. No LE com alterações do epitélio pigmentar da retina macular, os demais ficaram sem novidades clínicas. Tópicos (diclofenaco 0,1\% $($ ), (dorzolamida $2 \%+$ timolol $0,5 \%$ ( $)$ e espironolactona $50 \mathrm{mg}$ por via oral ao dia foram iniciados em todos os pacientes. Conclusões: A CSC ainda é uma condição enigmática devido em grande parte a uma história natural de melhora espontânea em uma grande proporção de pacientes. Nenhum tratamento isolado forneceu evidências esmagadoras de eficácia em ensaios clínicos randomizados publicados. Com o maior boom da era da pandemia COVID-19, como sua patogênese está relacionada ao estresse, favoreceria o agravamento da clínica.

Palavras-chave: Corioretinopatia Serosa Central, Descolamento Seroso de Retina, Covid-19, Dorzolamida, Timolol, Diclofenaco, Espironolactona. 


\section{Introducción}

La coriorretinopatía serosa central (CSC) es un trastorno idiopático caracterizado por desprendimiento de retina seroso y desprendimiento del epitelio pigmentario de retina (DEP). Los cambios se limitan con mayor frecuencia a la mácula y se relacionan con la fuga de líquido a través del epitelio pigmentario retiniano (EPR) hacia el espacio subretiniano. Los pacientes con CSC a menudo experimentan pérdida de la visión central, escotoma central, micropsia, metamorfopsia, disminución de la visión del color y anomalías en la sensibilidad al contraste. La agudeza visual puede reducirse solo moderadamente y puede haber un cambio hipermetrópico. ${ }^{1}$

Se han asociado numerosos factores de riesgo con la CSC, siendo el más constante el uso de glucocorticoides. Dada la fuerte asociación entre CSC y esteroides, su uso debe evitarse siempre que sea posible. Además, los pacientes con CSC deben ser interrogados sobre el uso de todas las formas de esteroides, incluidos los productos que pueden contener esteroides (p. Ej., Cremas para la piel, inyecciones articulares, aerosoles nasales, inhalantes y otras formas de glucocorticoides que comúnmente se pasan por alto), ya que estos podrían ser factores contribuyentes. ${ }^{2}$

El embarazo es un factor de riesgo reconocido de CSC. Los niveles de cortisol plasmático se elevan durante el embarazo, especialmente durante el tercer trimestre. La CSC asociada al embarazo tiende a presentarse como una exudación subretiniana blanca que generalmente se resuelve espontáneamente después del parto. ${ }^{3}$

Otro factor de riesgo tradicionalmente asociado con CSC es el estrés psicológico y la personalidad tipo A. ${ }^{7}$ Otras asociaciones incluyen hipertensión arterial sistémica, enfermedad por reflujo gastroesofágico y el uso de alcohol o agentes simpaticomiméti- cos, aunque esto último requiere una mayor confirmación. ${ }^{4-6}$

\section{Objetivo}

Demostrar la fluctuación clínica de 3 pacientes con coriorretinopatía serosa central crónica.

\section{Métodos}

Reporte de casos observacionales de coriorretinopatía serosa central crónica, en un período de seguimiento de noviembre 2019abril 2020. Se incluyeron las variables: revisión de historia clínica, fotografías, tomografía de coherencia óptica de dominio espectral y angiografía fluoresceínica.

\section{Resultados}

\section{CASO № 1.}

Masculino de 46 años de edad, procedente de Venezuela, con antecedentes familiares de 2 hermanos con secuelas CSC y una tía materna con secuelas crónicas CSC, usuario de antidepresivo tricíclicos y síntomas de atopia en la infancia con automedicación de esteroides, no precisando última dosis. Presentando pérdida repentinamente de agudeza visual en OD, metamorfopsia y escotoma central positivo. Después del primer episodio, persistió la disminución de la visión después de un periodo de recuperación del tratamiento médico subyacente.

\section{Examen clínico}

En el examen inicial, el paciente tenía Agudeza visual mejor corregida (AVMC) OD. 20/20 Ol 20/32 y PIO OD. 16 mmHg Ol 14 $\mathrm{mmHg}$. La biomicroscopia del segmento anterior de aspecto normal en ambos ojos. La oftalmoscopia del OD se observó un área redonda localizada de líquido subretiniano y el OI no mostró ningún cambio patológico. (Fig. 1A y 1B) 


\section{Exámenes complementarios OCT}

EI OCT en el OD se observó un desprendimiento neurosensorial seroso subfoveal,
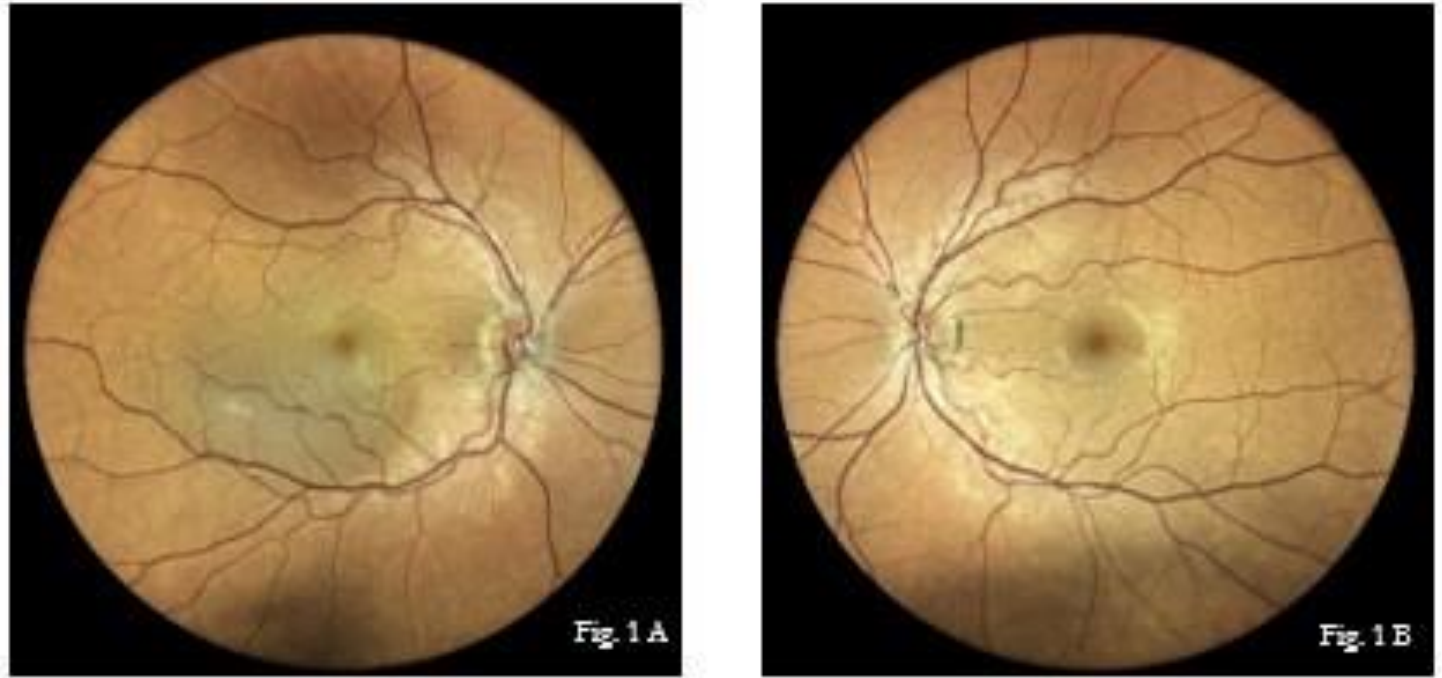

Fig. 1A. Imagen del fondo OD mostrando un área redonda de fluido subfoveal. Fig.1B. Ol Normal.
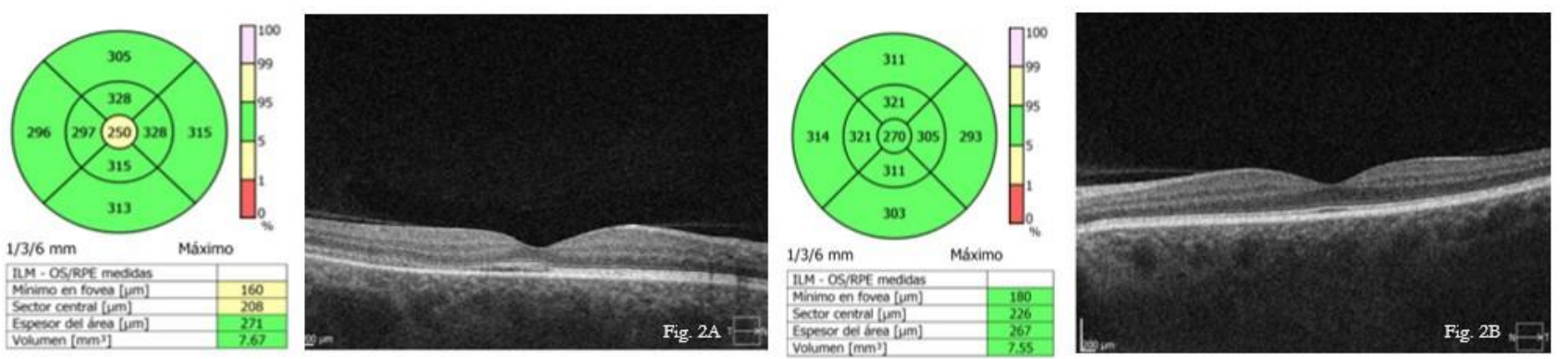

Fig. 2A. En OD se representa desprendimiento neurosensorial seroso subfoveal.

Fig. 2B. Ol normal.

Después de estos exámenes clínicos y paraclínicos, el diagnostico de CSC en el OD se confirmó y se inició el tratamiento con medicamentos tópicos antiinflamatorios no esteroides (diclofenaco $0.1 \% \AA 3$ veces al día, inhibidor de la anhidrasa carbónica más betabloqueantes no selectivos (dorzolamida $2 \%$ + timolol $0.5 \%$ (B) 2 veces al día y antagonistas de los receptores de mineralocorticoides (espironolactona $50 \mathrm{mg} \circledast$ ) vía oral diariamente.
En el segundo mes de seguimiento el OD. La OCT demostró la remisión parcial del desprendimiento seroso neurosensorial y con agudeza de 20/32. Se decide iniciar la terapia con espironolactona $50 \mathrm{mg} 1 \mathrm{vez}$ al día, con monitorización de los niveles de potasio en suero y el nivel de presión arterial. A los 6 meses de tratamiento de seguimiento, la AVMC se mantiene a 20/32 por periodo de un año, pero cuando interrumpió el tratamiento, la enfermedad recayó. 

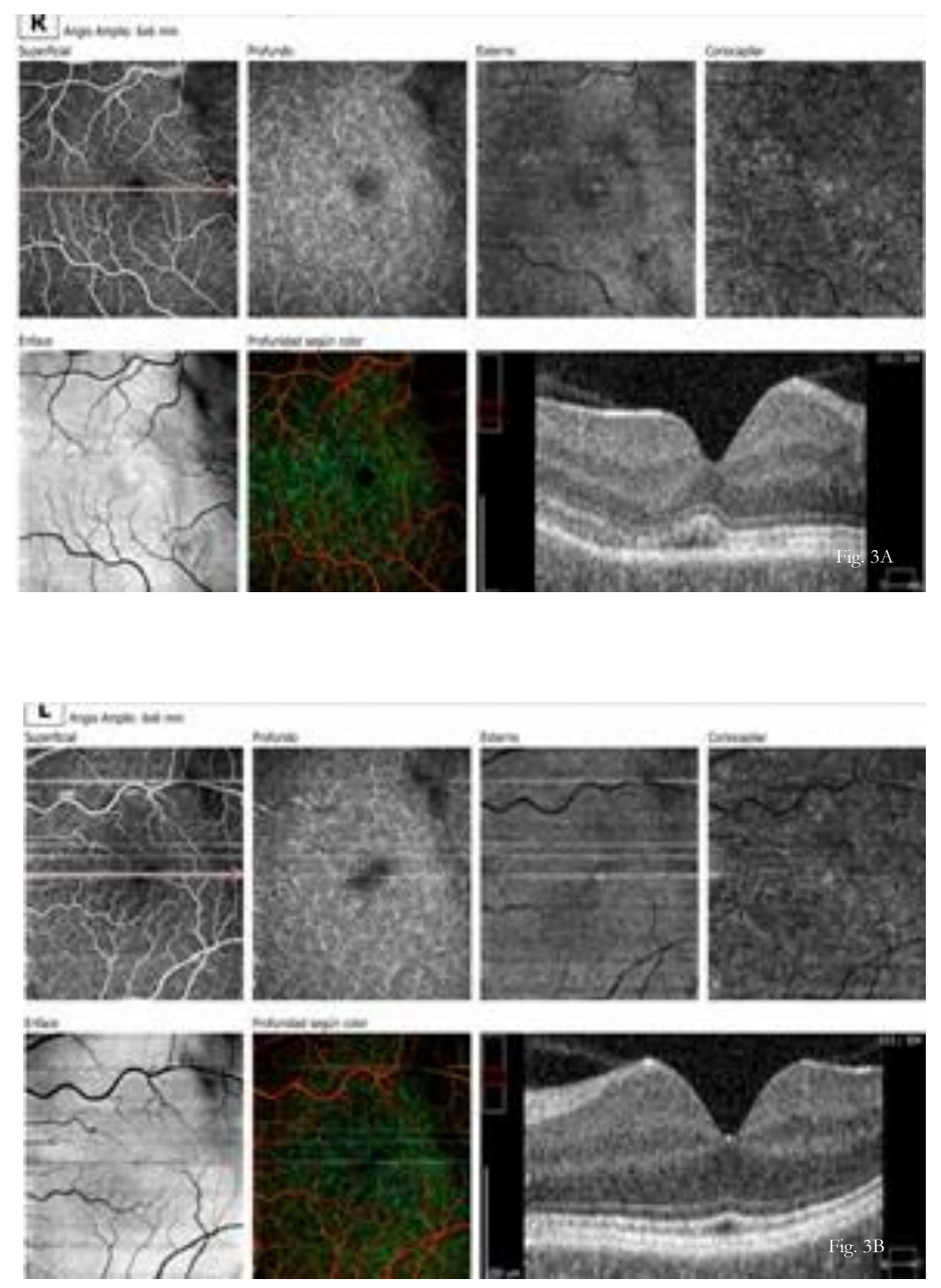

Fig. 3A. En OD se representa desprendimiento neurosensorial seroso subfoveal. Fig. 3B. OI normal

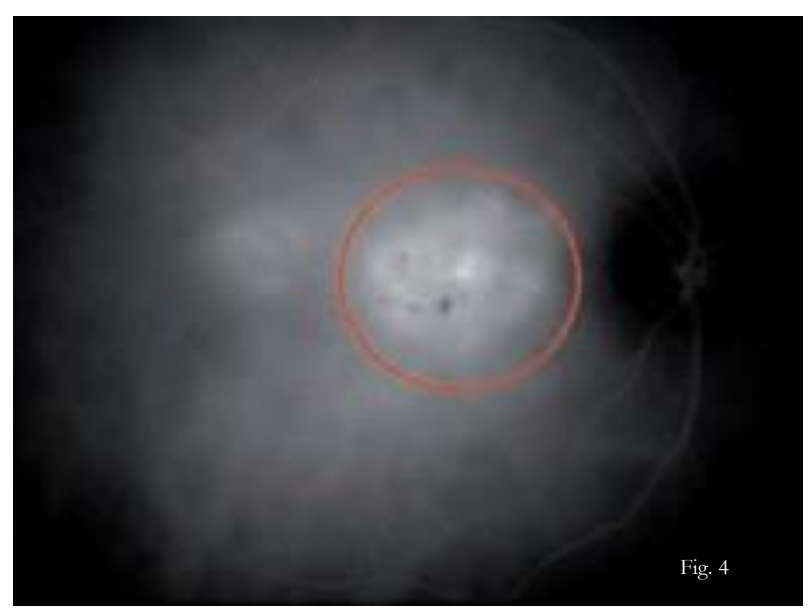

Fig. 4. Angiografía fluoresceínica. En OD presenta Hiperfluorescencia por acúmulo en área macular. 


\section{CASO № 2.}

Masculino de 53 años de edad, procedente de localidad, con antecedentes personales atópicos, marcado estrés laboral, personalidad tipo A. Presentando disminución repentinamente de agudeza visual en OI, metamorfopsia y escotoma central positivo acude a centro privado prescribiéndosele terapia esteroidea tópica y vía oral sin mejoría aparente. Después del primer episodio, continuó la disminución de la visión después de omitir dicho tratamiento e indicar

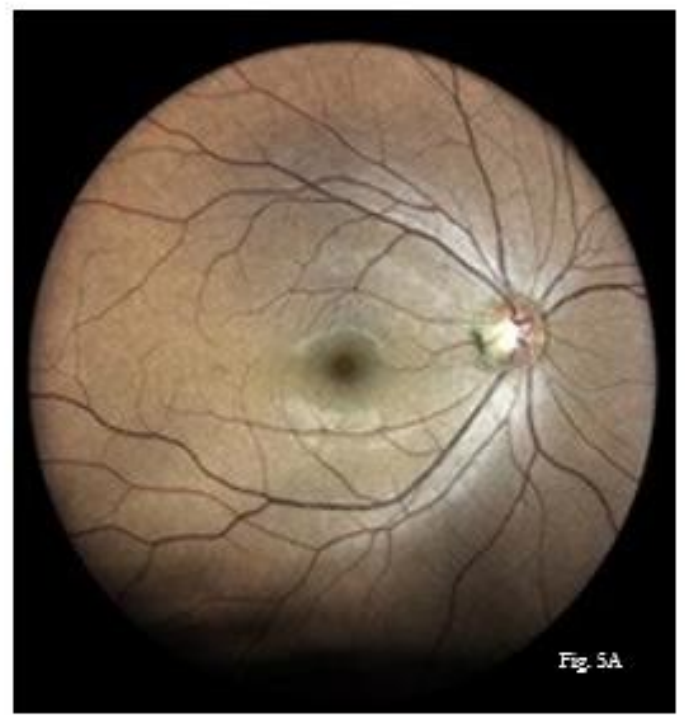

nueva medicación.

\section{Examen clínico}

En el examen inicial, la AVMC OD 20/25 y OI 20/50 y PIO 12 mmHg AO. La biomicroscopia del segmento anterior de aspecto normal en ambos ojos.

La oftalmoscopia del OD se observó dentro de límites normales y el Ol un área redonda macular localizada de líquido subretiniano.

(Figura. 5A y $5 B$ )

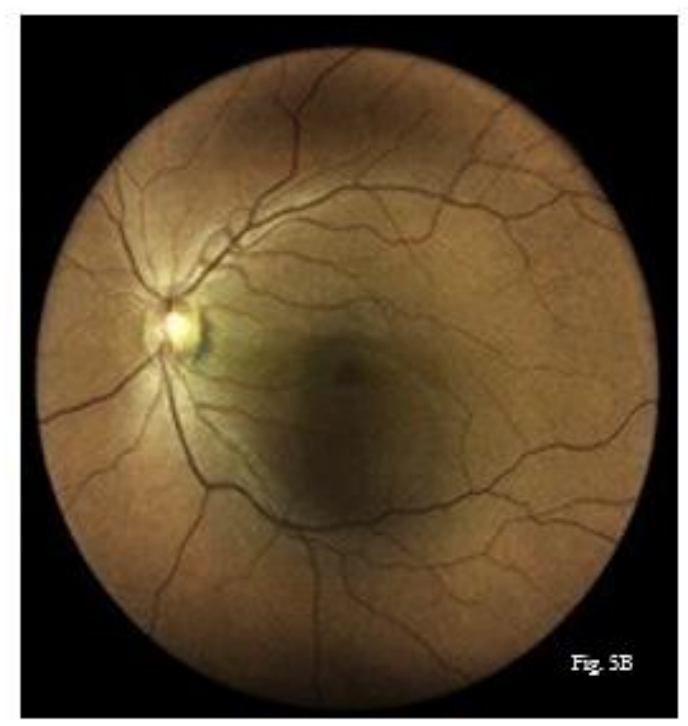

Fig.5A. OD normal. Fig.5B. Imagen del fondo OI mostrando un desprendimiento de retina neurosensorial seroso subfoveal.
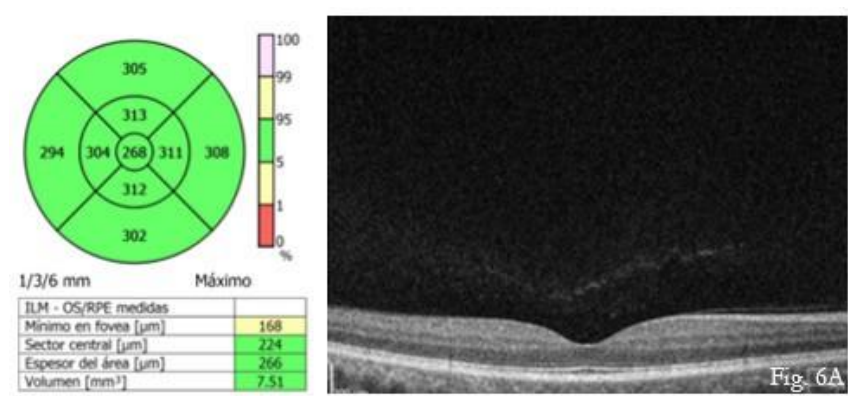

El OCT en el OD no mostró ningún cambio, Fig.6A. pero el ojo izquierdo representa un
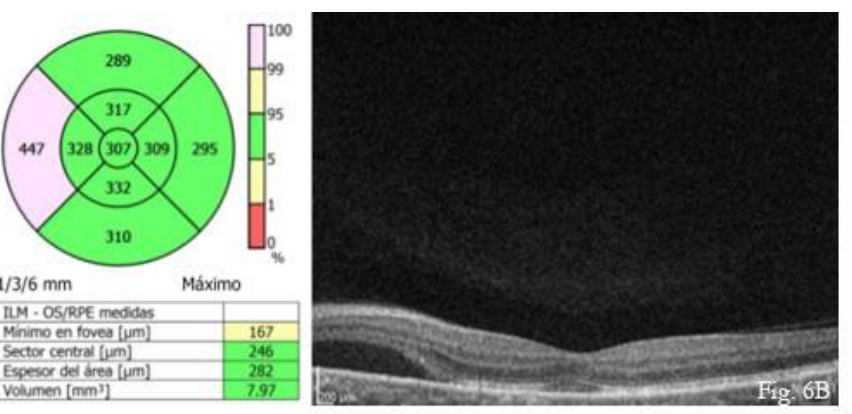

desprendimiento seroso subfoveal de retina neurosensorial Fig.6B.

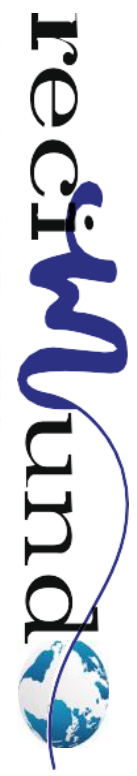




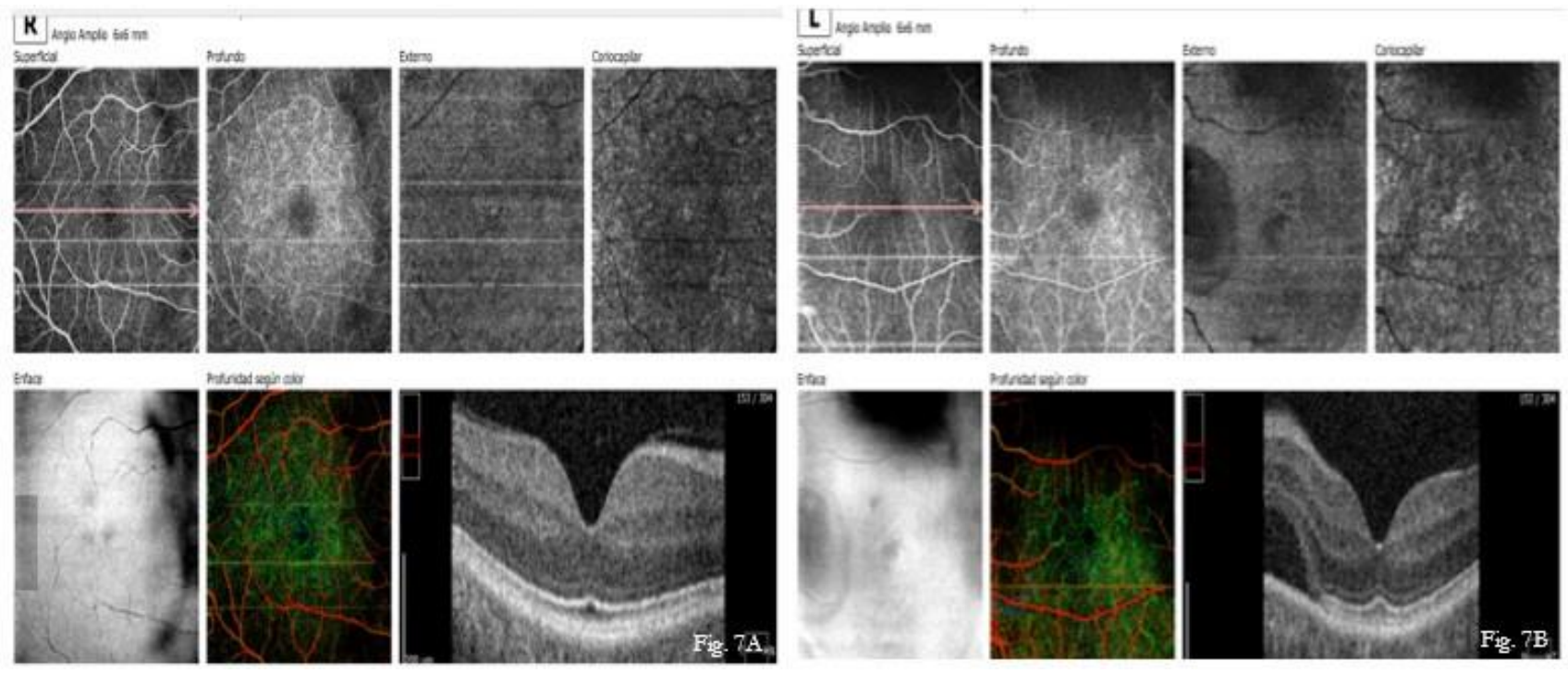

Fig. 7A. Angio OCT. OD Normal. Fig. 7B. Ol se evidencia DNS parafoveolar nasal.

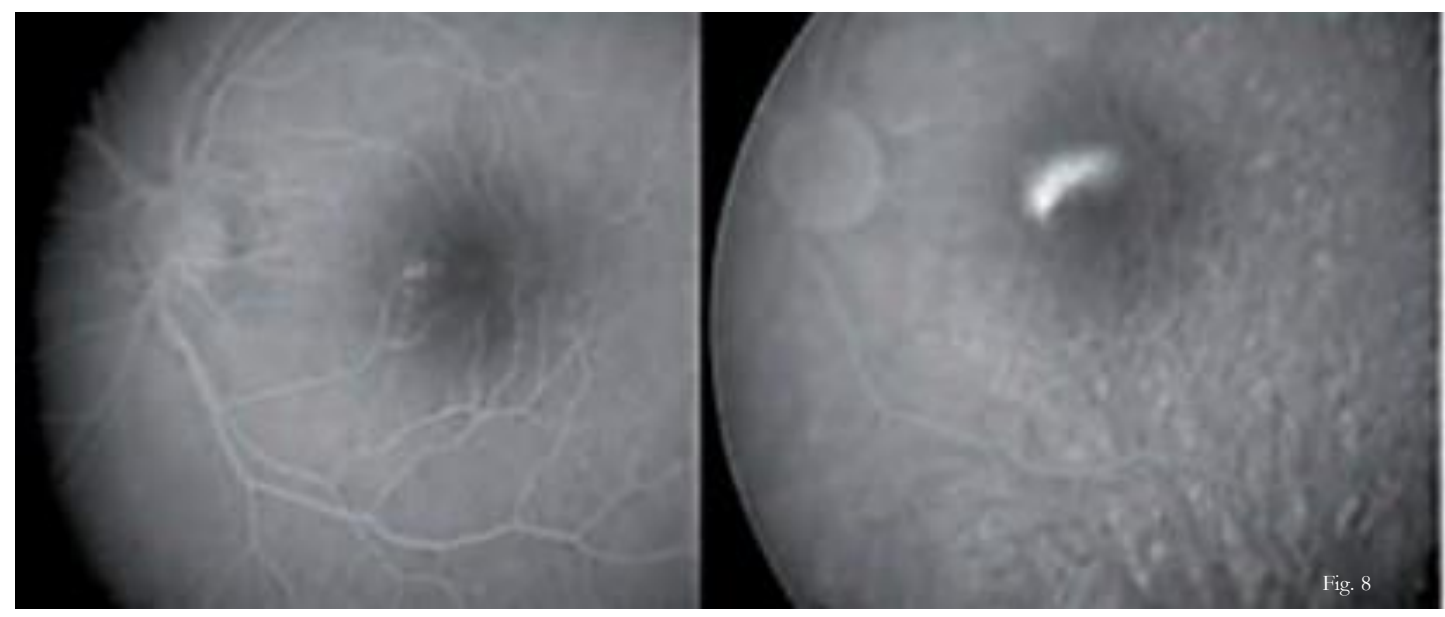

Fig. 8. Angiografía fluoresceínica. En Ol presenta Hiperfluorescencia por acúmulo en área parafoveolar nasal.

Después de estos exámenes clínicos y paraclínicos, el diagnostico de CSC en el OI se confirmó y se inició el tratamiento con medicamentos tópicos antiinflamatorios no esteroides (diclofenaco $0.1 \% \AA$ ) 3 veces al día; inhibidor de la anhidrasa carbónica más betabloqueantes no selectivos (dorzolamida $2 \%$ + timolol $0.5 \% @) 2$ veces al día y antagonistas de los receptores de mineralocorticoides (espironolactona $50 \mathrm{mg}{ }^{\circledR}$ ) vía oral diariamente.

En el segundo mes de seguimiento, el Ol. La OCT demostró la remisión parcial del desprendimiento seroso y el paciente tenía una agudeza de 20/32 en el Ol. Se decide mantener la terapia con espironolactona 50 mg una vez al día, con monitorización de los niveles de potasio en suero y el nivel de presión arterial; la AVMC se mantiene a 20/32 por periodo de 4 meses.

\section{CASO № 3.}

Masculino de 40 años de edad, procedente de localidad, con patrón de personalidad tipo A. Presenta disminución repentinamen- 
te de agudeza visual en OI, micropsia, metamorfopsia y escotoma central positivo de 7 meses de evolución. Después del primer episodio, continuó con mejoría de la visión después de dicho tratamiento e indicársele nueva medicación.

\section{Examen clínico}

En el examen inicial, la paciente tenía AVMC

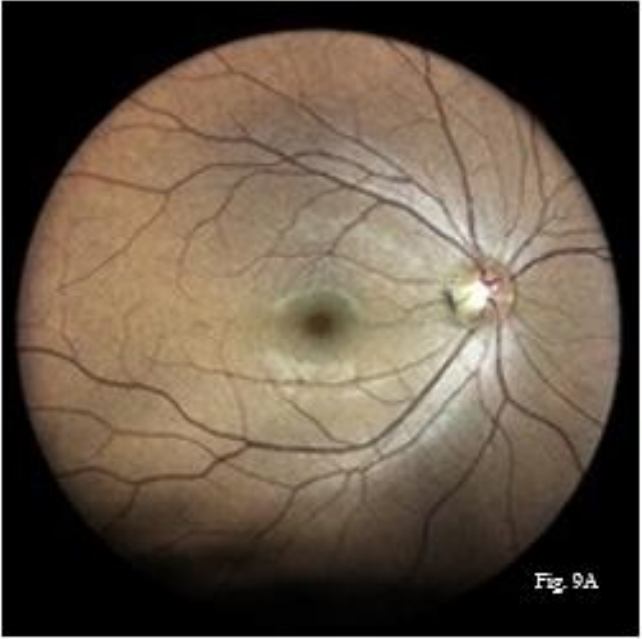

Fig.9A. OD normal.
OD. $20 / 20$ OI. $20 / 25$ y PIO OD. $11 \mathrm{mmHg}$ OI $12 \mathrm{mmHg}$. La biomicroscopia del segmento anterior de aspecto normal en ambos ojos.

La oftalmoscopia del OD se observó dentro de límites normales. (Fig. 9A). OI un área redonda macular localizada de líquido subretiniano. (Fig. 9B).

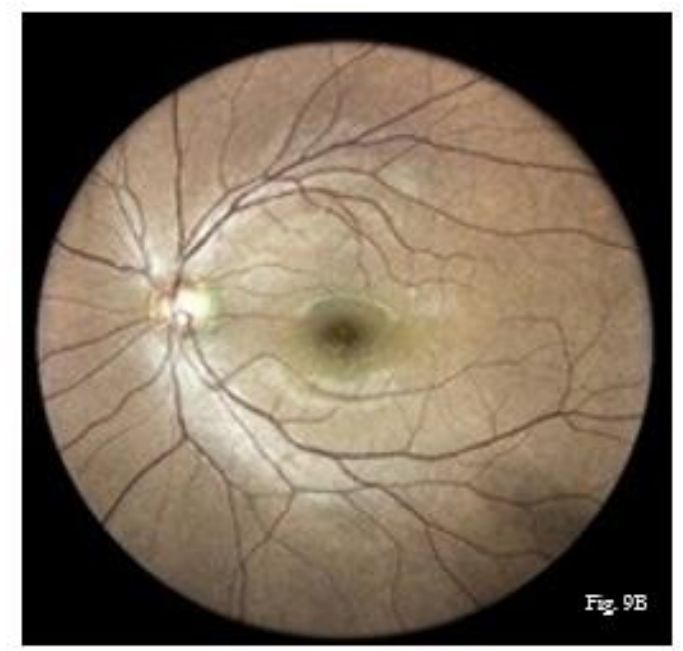

Fig. 9B. Imagen del fondo OI mostrando un área redonda macular localizada de líquido subretiniano.
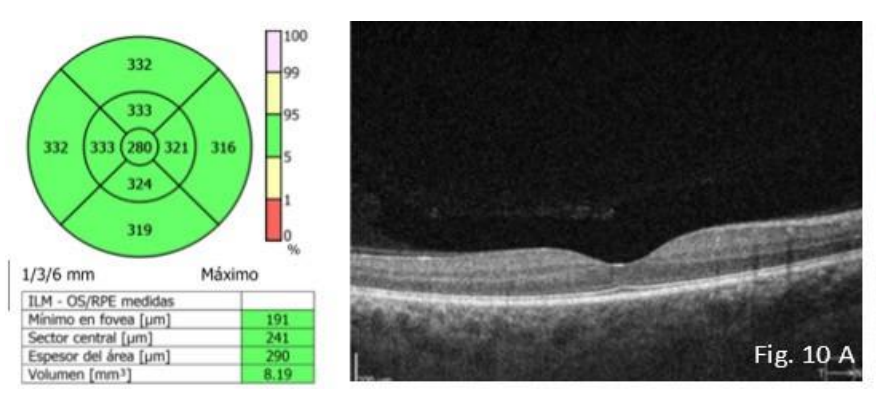

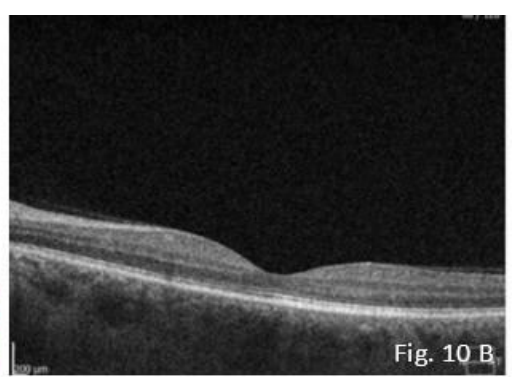

Fig. 10B
El OCT en el OD no mostró ningún cambio. Fig.10A.

Ol representa disrupción de epitelio pigmentario externo e internos de los fotorreceptores)

Fig. 10B.

Después de estos exámenes clínicos y paraclínicos, el diagnostico de CSC en el OI se confirmó y se inició el tratamiento con medicamentos antiinflamatorios no esteroides (tópicos) (diclofenaco $0.1 \% ®) ~ 3$ veces al día, inhibidor de la anhidrasa carbónica más betabloqueantes no selectivos (tópicos) (dorzolamida $2 \%+$ timolol $0.5 \% \circledast$ ) 2 veces al día y antagonistas de los recepto- res de mineralocorticoides (espironolactona 50 $\left.\mathrm{mg}{ }^{\circledR}\right)$ vía oral diariamente. 

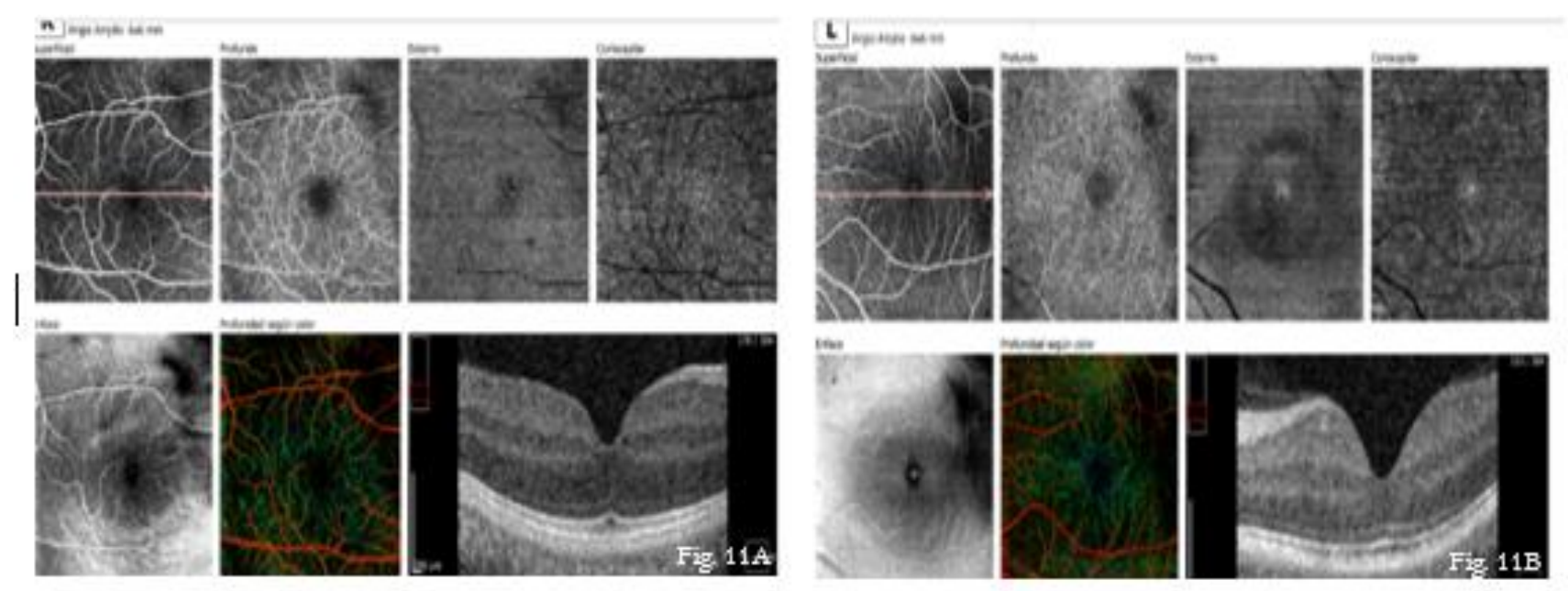

Fig. 11A. Angio OCT, OD normal. Fig. 11B. OI. Se representa disrupción de epitelio pigmentario retiniano (zona elipsoide a nivel de segmentos externo e internos de los fotorreceptores).

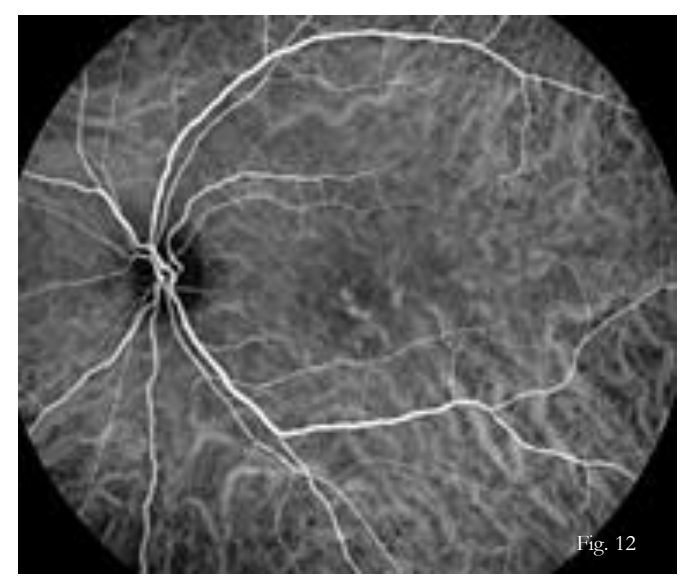

Fig. 12. Angiografía fluoresceínica. En Ol presenta Hiperfluorescencia por efecto de ventana en área macular.

Acude al séptimo mes de evolución. La OCT demostró la remisión total del desprendimiento seroso y el paciente tenía una agudeza de 20/25 en el Ol. Se decide mantener la terapia con espironolactona $50 \mathrm{mg}$ vía oral una vez al día, con monitorización de los niveles de potasio en suero y el nivel de presión arterial; la AVMC se mantiene a 20/25.

\section{Discusión}

La CSC aguda es típicamente un proceso autolimitado y su fisiopatología es poco co- nocida. Algunos postulan que un aumento focal en la permeabilidad de la coriocapilar abruma al EPR suprayacente, produciendo DEP serosos y líquido subretiniano. ${ }^{7}$ Guyer y col. Sugirieron que la patogenia de la CSC puede ser la hiperpermeabilidad vascular coroidea con o sin fugas epiteliales pigmentarias activas asociadas o DEP. Una teoría alternativa sugiere que la CSC es el resultado de una disfunción de la bomba de iones en el EPR, lo que resulta en un movimiento inverso del líquido en una dirección coriorretiniana. $^{8}$ 
La recuperación de la agudeza visual ocurre típicamente dentro de 1 a 4 meses, coincidiendo con la reinserción de la retina neurosensorial, con pocas secuelas visuales reconocidas. Las recurrencias son comunes y ocurren en aproximadamente el $30 \%$ al $50 \%$ de los pacientes al año. ${ }^{9}$ Los pacientes con recurrencias frecuentes o con desprendimiento de retina neurosensorial crónico pueden desarrollar atrofia del EPR y cambios de retina neurosensoriales que resultan en una pérdida permanente de la visión funcional. En esta discusión se utilizarán los términos agudo y crónico (líquido que persiste $>3$ meses).

La observación es el tratamiento inicial estándar en pacientes con CSC aguda, pero hay casos en los que el tratamiento puede ser deseable. Estos casos incluyen CSC con líquido subretiniano macular persistente (SRF) o agudeza visual reducida, casos en los que se requiere una recuperación rápida de la visión por razones vocacionales y aquellos en los que CSC no tratada ha dado como resultado un resultado visual deficiente en el otro ojo.

La fotocoagulación con láser focal se usa comúnmente para acelerar la absorción de SRF en CSC aguda y crónica. Normalmente, las quemaduras con láser se aplican a áreas de fuga focal identificadas por angiografía con fluoresceína (AF) como las principales fuentes de SRF. El tratamiento de estas fugas suele conducir a la resolución de la SRF; sin embargo, en raras ocasiones, la fotocoagulación con láser puede estar asociada con un escotoma persistente en el sitio de la fotocoagulación y la neovascularización coroidea (NVC). Por lo tanto, no se recomienda la fotocoagulación con láser si el punto de fuga está dentro o cerca de la zona avascular foveal. ${ }^{10}$

Otros tipos de administración de láser también pueden ser eficaces en el tratamiento de CSC. El modo de administración deláser de micropulso disminuye el riesgo de daño térmico iatrogénico porque no induce quemaduras visibles por láser $\mathrm{y}$, por lo tanto, puede utilizarse para tratar fugas focales y difusas subfoveales o yuxtafoveales. ${ }^{11} \mathrm{La}$ terapia fotodinámica (TFD) con el fármaco activado por luz verteporfina (Visudyne, Bausch \& Lomb) se ha utilizado eficazmente para tratar la CSC crónica. Las ventajas potenciales del láser de micropulso sobre la TFD incluyen un menor costo y la ausencia de eventos adversos asociados con la infusión de TFD (p. Ej., Reducción transitoria de la función macular, falta de perfusión coroidea, atrofia del EPR y NVC).

Roca y col. Llevaron a cabo un estudio comparativo retrospectivo multicéntrico de 159 ojos consecutivos con CSC crónica tratados con láser de micropulso amarillo o TFD con verteporfina de media dosis. ${ }^{12}$ Las principales medidas de resultado fueron la AVMC y el grosor macular central (GMC) a los 12 meses. Se consideró estadísticamente significativo un valor de probabilidad inferior a 0,05 .

Se han investigado innumerables medicamentos sistémicos como tratamientos para la CSC, incluida la rifampicina, el tratamiento triple de helicobacter pylori, los inhibidores de la anhidrasa carbónica, el finasteride, los betabloqueantes, los antioxidantes y la aspirina. En conjunto, estos han tenido malos resultados. ${ }^{13}$

Los antagonistas de los receptores de mineralocorticoides parecen ser eficaces en pacientes con CSC crónica o recurrente, demostrando una mejoría anatómica y funcional. Bousquet y col. Compararon espironolactona $50 \mathrm{mg}$ al día durante 30 días versus placebo en pacientes con SRF persistente durante al menos 3 meses. Así mismo encontraron una reducción estadísticamente significativa en la SRF y una reducción del grosor coroideo subfoveal en los ojos tratados con espironolactona en comparación con el placebo. No se observaron cambios significativos en la AVMC y 
no se informaron complicaciones relacionadas con el tratamiento. ${ }^{14}$

La espironolactona, un antagonista selectivo del receptor de aldosterona y diurético ahorrador de potasio que fue aprobado para el tratamiento de la hipertensión arterial resistente, recientemente demostró que mejora la agudeza visual y disminuye significativamente el grosor macular central en una pequeña serie de pacientes con CSC crónica. ${ }^{14,16}$ El medicamento generalmente se tolera bien, pero las interacciones farmacológicas deben descartarse antes del inicio y el potasio sérico y la presión arterial deben controlarse durante el tratamiento. A pesar de que cuenta con algunos efectos adversos como lo son: ginecomastia, trastornos menstruales, atrofia testicular, ataxia cerebral, disfunción eréctil, somnolencia, rash cutáneo. Se están realizando estudios más grandes, prospectivos, controlados con placebo para investigar más a fondo la eficacia de esta opción de tratamiento.

Schwartz y col. Compararon la eficacia y seguridad del antagonista de la aldosterona eplerenona $50 \mathrm{mg} /$ día en pacientes con CSC crónica versus placebo. La principal medida de resultado fue el cambio en la SRF a los 3 meses. Los resultados secundarios incluyeron el grosor coroideo y los cambios en la MAVC. En este estudio, la eplerenona no fue superior al placebo. ${ }^{15}$ Se sabe que la espironolactona tiene una mayor afinidad por el receptor de mineralocorticoides que la eplerenona, junto con una mayor incidencia de efectos secundarios, que incluyen hiperpotasemia, ginecomastia, disminución de la libido y cambios menstruales. ${ }^{13}$

Los agentes anti-VEGF no se consideran tratamientos de primera línea para CSC aguda o crónica porque los niveles acuosos de VEGF no están elevados en estos pacientes. Sin embargo, la terapia anti-VEGF es útil en pacientes en los que se desarrolla NVC secundaria. ${ }^{16}$

\section{Conclusión}

El estrés negativo durante la pandemia ha provocado un aumento de un $15 \%$ de las patologías oculares entre ellas la CSC. La sociedad se ha enfrentado a una situación completamente nueva a causa del coronavirus. Miedo al contagio del COVID-19, ansiedad durante el confinamiento, tensión y preocupación por amigos y familiares enfermos, los problemas económicos derivados del parón de actividad. El manejo de la CSC es un desafío, particularmente la CSC crónica. Aunque el láser focal y la TFD son los estándares actuales de atención en la CSC crónica, el láser de micropulso es una buena alternativa en los casos en que no se dispone de verteporfina. En nuestra serie de casos, el tratamiento con espironolactona se asoció con una reducción significativa en el nivel de líquido subretiniano y una mejora en la agudeza visual, pero solo dependiente de la dosis. La interrupción del tratamiento indujo una recaída de la enfermedad.

\section{Bibliografía}

1. Wang M, Munch Ic, Hasler Pw, Prünte C, Larsen M. Central serous chorioretinopathy. Acta ophthalmol (copenh). march 2008;86(2):126-45.

2. Yanoff $M$, Duker Js. Ophthalmology printbook, 2013, ed. Elsevier.

3.Chumbley Lc, Frank Rn. Central serous retinopathy and pregnancy. Am j ophthalmol. February de 1974;77(2):158-60.

4. Haimovici R, Koh s, Gagnon Dr, Lehrfeld T, Wellik $S$, central serous chorioretinopathy case-control study group. Risk factors for central serous chorioretinopathy: a case-control study. ophthalmology. February 2004;111(2):244-9.

5.Michael Jc, Pak J, Pulido J, De Venecia G. central serous chorioretinopathy associated with administration of sympathomimetic agents. Am j ophthalmol. July 2003;136(1):182-5.

6.Mansuetta Cc, Mason Jo, Swanner J, Feist Rm, White Mf, Thomley Ml, et al. An association between central serous chorioretinopathy and gastroesophageal reflux disease. Am j ophthalmol. June 2004;137(6):1096-100. 
7.Gemenetzi M, De salvo G, Lotery Aj. Central serous chorioretinopathy: an update on pathogenesis and treatment. eye lond engl. December 2010;24(12):1743-56.

8.Guyer Dr, Yannuzzi La, Slakter Js, Sorenson Ja, Ho A, Orlock D. Digital indocyanine green videoangiography of central serous chorioretinopathy. Arch ophthalmol chic ill 1960. August 1994;112(8):1057-62.

9.Klein MI, Van Buskirk Em, Friedman E, Gragoudas E, Chandra S. Experience with nontreatment of central serous choroidopathy. arch ophthalmol chic ill 1960. April 1974;91(4):247-50.

10. Nicholson B, Noble J, Forooghian F, Meyerle C. Central serous chorioretinopathy: update on pathophysiology and treatment. surv ophthalmol. April 2013;58(2):103-26.

11.Sivaprasad S, Elagouz M, Mchugh D, Shona O, Dorin G. Micropulsed diode laser therapy: evolution and clinical applications. surv ophthalmol. December 2010;55(6):516-30.

12.Roca Ja, Wu L, Fromow-Guerra J, Rodríguez Rj, Berrocal Mh, Rojas S, et al. Yellow (577 nm) micropulse laser versus half-dose verteporfin photodynamic therapy in eyes with chronic central serous chorioretinopathy: results of the pan-american co-
Ilaborative retina study (pacores) group. br j ophthalmol. 2018;102(12):1696-700.

13.Delyani Ja. Mineralocorticoid receptor antagonists: the evolution of utility and pharmacology. kidney int. April 2000;57(4):1408-11.

14.Bousquet E, Beydoun T, Rothschild P-R, Bergin C, Zhao M, Batista R, et al. Spironolactone for nonresolving central serous chorioretinopathy: a randomized controlled crossover study. retina phila pa. December 2015;35(12):2505-15.

15.Dchwartz R, Habot-Wilner Z, Martinez Mr, Nutman A, Goldenberg D, Cohen S, et al. Eplerenone for chronic central serous chorioretinopathy-a randomized controlled prospective study. Acta ophthalmol (copenh). November 2017;95(7): e 610-8.

16.Denniston Ako, Murray Pi. Oxford rapid reference for ophthalmology, 2008, oxford university press, 420. American academy of ophthalmology, retina and vitreous, 2007-2008, 51-53.

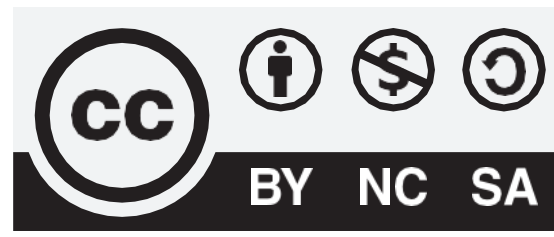

CREATIVE COMMONS RECONOCIMIENTO-NOCOMERCIAL-COMPARTIRIGUAL 4.0.

\section{CITAR ESTE ARTICULO:}

Barrientos Ortiz, R., \& González Méndez, A. L. (2020). Coriorretinopatía serosa central crónica. En época de pandemia por covid-19. Serie de casos. RECIMUNDO, 4(4). https://doi. org/10.26820/recimundo/4 (4). noviembre.2020.216-227 